"C2020 IEEE. Personal use of this material is permitted. Permission from IEEE must be obtained for all other uses, in any current or future media, including reprinting/republishing this material for advertising or promotional purposes, creating new collective works, for resale or redistribution to servers or lists, or reuse of any copyrighted component of this work in other works." 


\section{Proposing a Framework for Resilient Active Distribution Systems using Withstand, Respond, Adapt, and Prevent Element}

\author{
Dillip Kumar Mishra \\ School of Electrical and Data Engg. \\ University of Technology Sydney \\ Sydney, Australia \\ dkmishra@ieee.org
}

\author{
Mojtaba Jabbari Ghadi \\ School of Electrical and Data Engg. \\ University of Technology Sydney \\ Sydney, Australia \\ Mojtaba.JabbariGhadi@ \\ student.uts.edu.au
}

\author{
Li Li \\ School of Electrical and Data Engg. \\ University of Technology Sydney \\ Sydney, Australia \\ Li.Li@uts.edu.au
}

\author{
Jiangfeng Zhang \\ School of Electrical and Data Engg. \\ University of Technology Sydney \\ Sydney, Australia \\ Jiangfeng.Zhang@uts.edu.au
}

\begin{abstract}
The increasing frequency of natural disasters and man-made attacks have increased power outages worldwide. Thus, a resilient infrastructure must be constructed to reduce power system damages which directly impacts on the social and economic lives of people. In this paper, a new framework called withstand, respond, adapt, and prevent (WRAP) is presented to evaluate and improve the resilience of distribution networks following a review on existing studies. This resilience enhancement may happen through microgrid and multimicrogrid development in planning or operation stages. Each element of the WRAP framework is responsible for the improvement of the power system resilience in terms of its own attributes and resilience evaluation index. Furthermore, the WRAP framework is defined on the basis of a flowchart with respect to conditional statements. The WRAP framework can be a helpful solution in measuring the resiliency of the power system in terms of robustness, rapidity, adaptability, and predictability. Finally, a case study considering energy-notsupplied as a resilience evaluation index is presented.
\end{abstract}

Keywords-Active distribution system (ADS), power system resilience, withstand, recover, adapt and prevent (WRAP)

\section{INTRODUCTION}

The electric power system is considered as the backbone of modern society. Thus, its operation should be safe, reliable, and efficient to maintain stability in terms of social and economic aspects[1,2]. Although component failures occur due to atmospheric conditions or its haphazard nature, power systems are planned and designed on the basis of $\mathrm{N}-1$ security criteria to address power system vulnerability $[3,4]$. However, the frequency of natural disasters and man-made attacks has been increasing over the decades, thereby increasing power outages; this phenomenon indicates that the design is poorly equipped for extreme eventualities of a bulk power system and level of severity $[5,6]$. Therefore, preparing for unexpected events should be prioritized. Resilient power systems can continuously supply electricity even after the occurrence of natural disasters [7]. Understanding resilient power systems, specifically in terms of their risk, safety, and reliability is a major task [8-10]. In previous decades, several smart technologies have been used to enhance grid reliability and resilience, for example, smart grid, microgrid, and multimicrogrid. Resilient power systems are mainly focused on providing a reliable power supply to consumers during and after disrupted events [11]. Moreover, the increasing frequency of disruptive extreme can result in the loss of nonrobust electricity infrastructures and components that are directly related to critical losses in economy, information technology-related services, water supply, medical services, and security. Obviously, these services cannot be executed without electricity. Hence, several aspects must be considered in the design of electrical infrastructures, and the existing system must be renovated and transformed into a robust one to withstand a large range of possible events . Fig. 1 presents the number of people affected by power outages from 1999 to 2019 across different countries. Fig. 2 shows the various events from 1999-2019 that have caused these power outages. As can be seen, the major percentage of power outages is caused by equipment failures and natural disasters [12].

On the other hand, the world has observed the stunning transformations of electrical infrastructures in terms of reliability, sustainability, and resiliency over the past few decades. This perception led to the "light on" facility system design that already possesses reliable and sustainable grids and is now aiming at reaching a resilient one [13]. While a reliable grid pertains to the light on phenomenon during normal operating conditions, sustainable grid refers to meeting the necessities of current scenarios without conceding the capability of future energy market to meet the specific requirements. Beside, resilient grid refers to the notion of keeping the light on during and after highly disruptive natural events. Noteworthy, such changes should happen in both aspects of planning and operation of the grid to reach a network which has the ability to maintain the continuity of supply after severe events.

Based on the above discussions, the power system resiliency is concerned with four main factors: withstand, respond, adapt, and prevent (WRAP) [14]. These factors are needed to be considered while designing resilient electrical infrastructures to effectively respond to natural disasters and other malicious attack scenarios. As far as the resilience timeframe is concerned, passively withstanding the disaster (i.e., before, during, and after) signifies the providence of uninterrupted power supply to emergency loads (i.e., the first responders like hospitals) regardless of outages in other regions.

In order to brighten the concepts associated with the operation of resilient power systems, this paper proposes a new framework that considers and discusses each individual component of WRAP, as well as its respective attributes. 


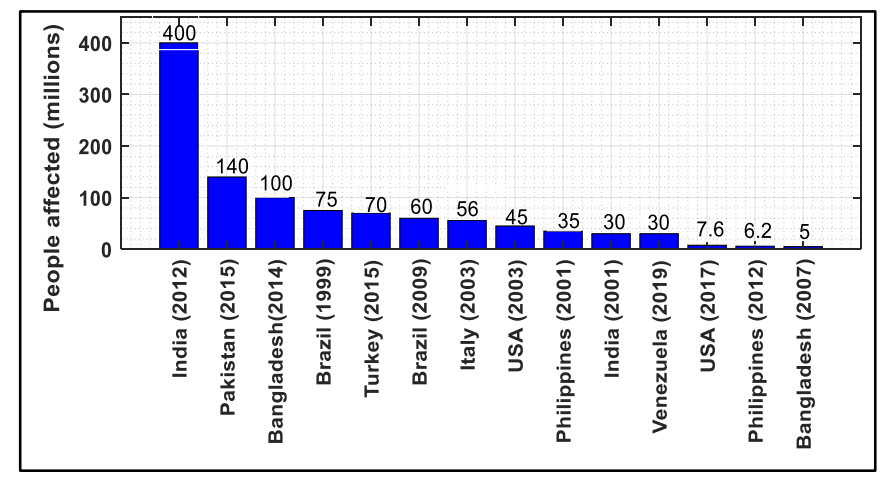

Fig. 1. Major Power outages

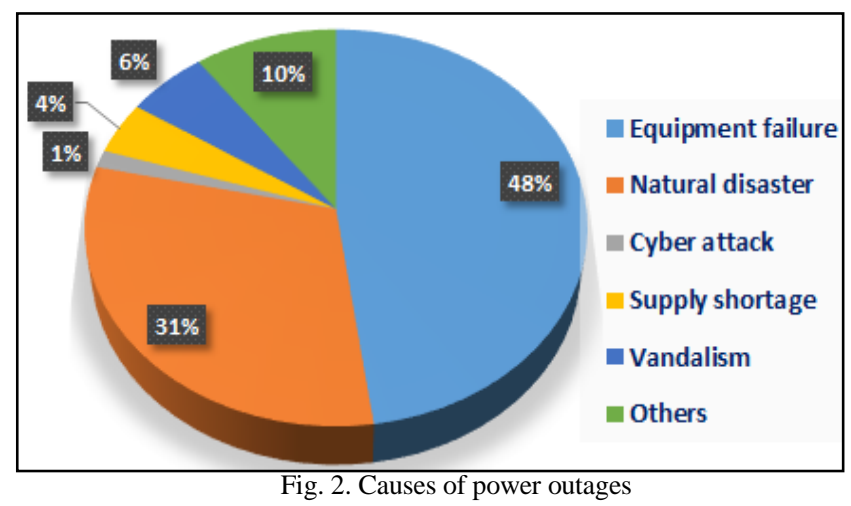

Fig. 2. Causes of power outages

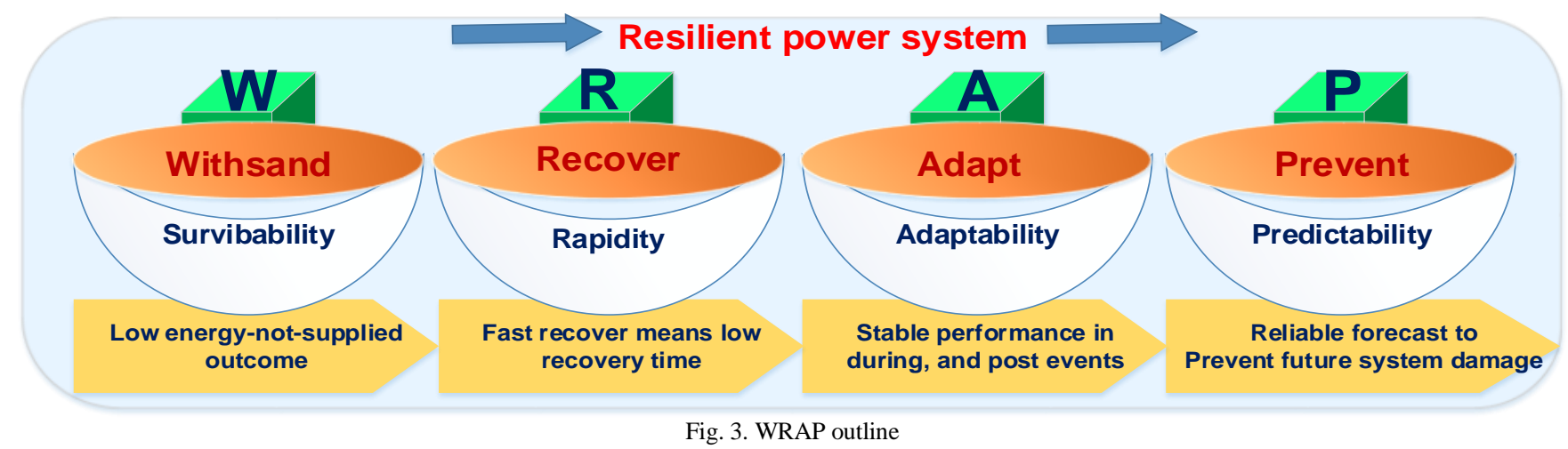

Moreover, a novel technique for the enhancement of power system resilience that can reduce the restoration period is also discussed. Furthermore, the factors associated with the WRAP model, as well as the model's resilience evaluation index, are defined.

\section{WRAP MODEL}

In the restructured power systems, power producers are migrating from bulk units to small-scale ones (e.g., microgrid and multi-microgrid). This type of structure helps to provide excellent energy management capabilities, improves the reliability of response to the disruptive events, and enhances resilience characteristics. As an illustration, microgrids are used to enhance the automated control of distribution systems under blackouts [15] and to meet the demands of external loads through the least switch operations. The self-healing strategy of networked microgrids with an economic dispatch plan was presented in [16], in which the surplus energies in each unit were accumulated to satisfy the power demands. Aside from energy restoration, microgrids can also contribute to international frequency regulation through supplementary control loops [17, 18]. Refs. [19-21] presented an in-depth analysis and discussion of the communication between coupled microgrids and distribution system operators.

Increasing attention has been focused on the extension of a single microgrid to interconnected microgrids to avert standalone failure and reduce communication overhead [22, 23]. In addition, a resilient power system is a current research trend. The WRAP elements need to be considered to enhance the severity of resilience, and a robust electrical infrastructure must be constructed to solve the issue in resilient characteristics. WRAP can lessen the vulnerability of power systems while maintaining its stability.

The concept of WRAP outline is depicted in Fig. 3 and each element of WRAP including withstand, recover, adapt and respond is shown in Fig. 4, Fig. 5, Fig. 6 and Fig. 7 respectively.

\section{A. Withstand}

Withstand is concerned with the preparedness, reinforcement, and robustness of the system against extreme events which are not considered in the traditional system design. Different risks, events, and hazards are estimated in terms of reliability. In addition, training and mandated maintenance programs must be conducted to improve the survivability and sustainability of the power system. Survivability indicates that power systems should withstand disruptive natural events with minimal damages, whereas sustainability refers to the continuity of energy supply to the end-users during and after the occurrence of extreme events [24, 25]. Moreover, skilled engineers should offer a series of intelligence exchange program regarding technological advancement and new protective measure schemes to improve resiliency. Some research studies have consistently shown that these factors emphasize the improvement of power system resiliency.

To measure the attributes of the withstand element, the system's redundancy, prevention, and key maintenance operation should be assessed. Moreover, to measure the significance of this element, "energy not supplied" [26] can be taken as a resilience evaluation index, which indicates the volume of energy to customers that are lost as a result of faults or failures on the network. The power system should be designed to cope with disruptive natural events and/or manmade attacks, which consequently minimizes the resilience evaluation index (e.g., energy not supplied) and enhances reliability and resiliency.

\section{B. Recover}

Power system recovery has been attracting increasing attention because of the significant dependence of all social 


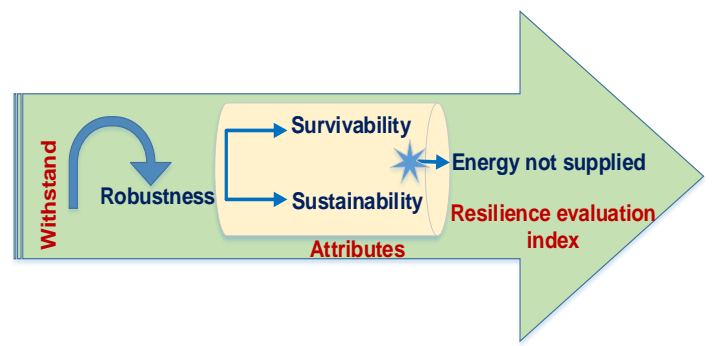

Fig. 4. Withstand phase

and communicating networks on electricity; so that a delay in electric power system recovery may lead to immense economic losses. Therefore, the recovery stage is concerned with the rapid restoration of a system [27]. Various mechanisms, such as reserve scheduling, black start, crew member deployment, on-site generation units, and island operations, are used to recover the power system [28, 29]. During black start, the grid can restore the operation without relying on superficial networks to recover from total or partial load curtailment. More crew member deployment is a good solution to decrease the restoration time and is also important for vulnerable components $[30,31]$. In the past decades, the number of on-site generation facilities has significantly increased to restore the distribution network and to provide load supply during emergencies. In this phase, two attributes are considered: rapidity and vulnerability. Rapidity refers to the speed of recovery, while vulnerability signifies the weak units of the system. Moreover, the resilience evaluation indices of this phase are time and cost of recovery; the effectiveness of the system is verified when the system is restored within a short time with low cost [27, 32-35].

\section{Adapt}

To adapt the distribution system to recognize the stabilized performance during, and after an event, one of the most significant schemes i.e. reconfiguration planning must be taken into account. Besides, numerous factors, including restructure, policy change, smooth functionality units, emerging hazard assessments, and periodic reviews should be considered in customizing the power system network [36-41]. These factors can reconcile the system against unfavorable events [42]. Nowadays, the power system reconfiguration is of importance due to the increasing number of disasters and man-made attacks. Small-scale power systems are now preferred over large-scale ones because microgrids, which is an example of the former, can reduce the total outage of the mainstream grid by means of self-healing mode. In addition, the enhancement of resilience indices has become a key economic factor that can reduce power system damage and recovery cost and provide reliable power supply to the endusers, which is crucial for distribution planning [43]. Several components, including energy storage units, on-site distributed generation units, smart transformers, and fault

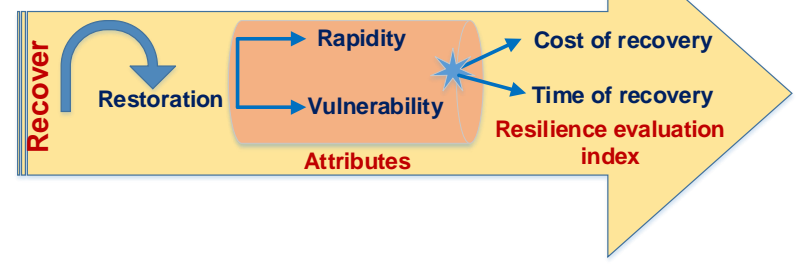

Fig. 5. Recover phase

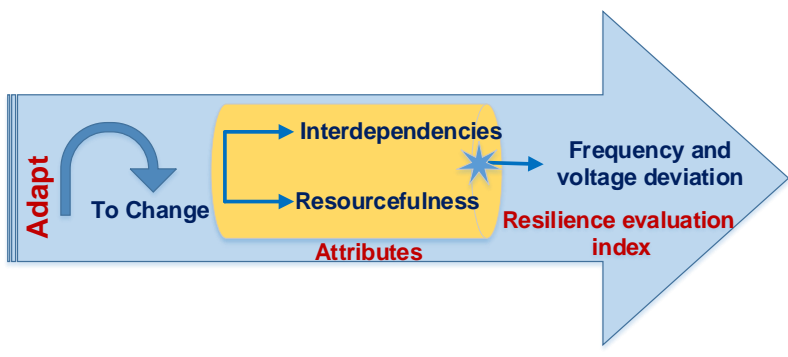

Fig. 6. Adapt phase

protection devices can be installed to reconfigure distribution systems. Furthermore, the integration of renewable energy sources and multiple microgrid formation are also parts of the reconfiguration phase.

Interdependency and resourcefulness are taken as the attributes of the adapt phase. However, the most distinct element of this phase is the controller (centralized/ decentralized) involving a minimization technique that can smoothly tune and mitigate the transients of the power system [44]. In addition, the frequency and voltage deviations can also play a crucial role during the events, and they have to be stabilized through controlling the renewable sources and flexible alternating current devices [45, 46]. More importantly, this phase signifies the stability of the system during the events, and eventually enhance the resilience.

\section{Prevent}

To prevent power system disruption, several measures, such as reliable forecasting, review of previous power outages, interventions, utilization of good quality components, and preparations for future attack scenarios, are considered [47, 48]. In addition, many different factors, such as identification of vulnerable units, an arrangement of protective units, decision-making tools, and risk reduction methods should be considered to precisely predict approaching events and prevent power system damages caused by disasters [49, 50]. A detailed disaster study has greatly influenced the assessment of attributes [51]. Considering the previous data, the upcoming catastrophe can be predicted, and the system should be prepared according to these scenarios. Thus, crew member deployment, on-site generation facilities, disconnection of less vulnerable components, and easy access to emergency loads must be planned properly. Several studies that investigate the pre-event strategies against disaster in the power system context have been carried out [52-55]. In this phase, the probability of failure can be considered as a resilience evaluation index to measure the consequences of extreme events in power systems [56]. This function signifies system damage or the major fault, which can be classified as permanent or temporary fault, depending on the weather intensity. The graph of the relationship between the probability of failure and weather intensity is known as the fragility curve.

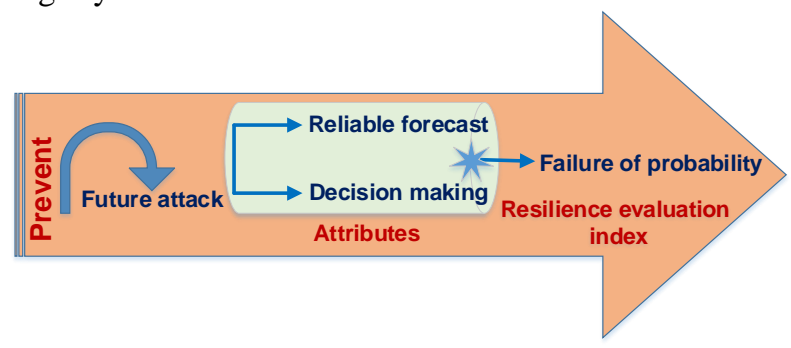

Fig. 7. Prevent phase 


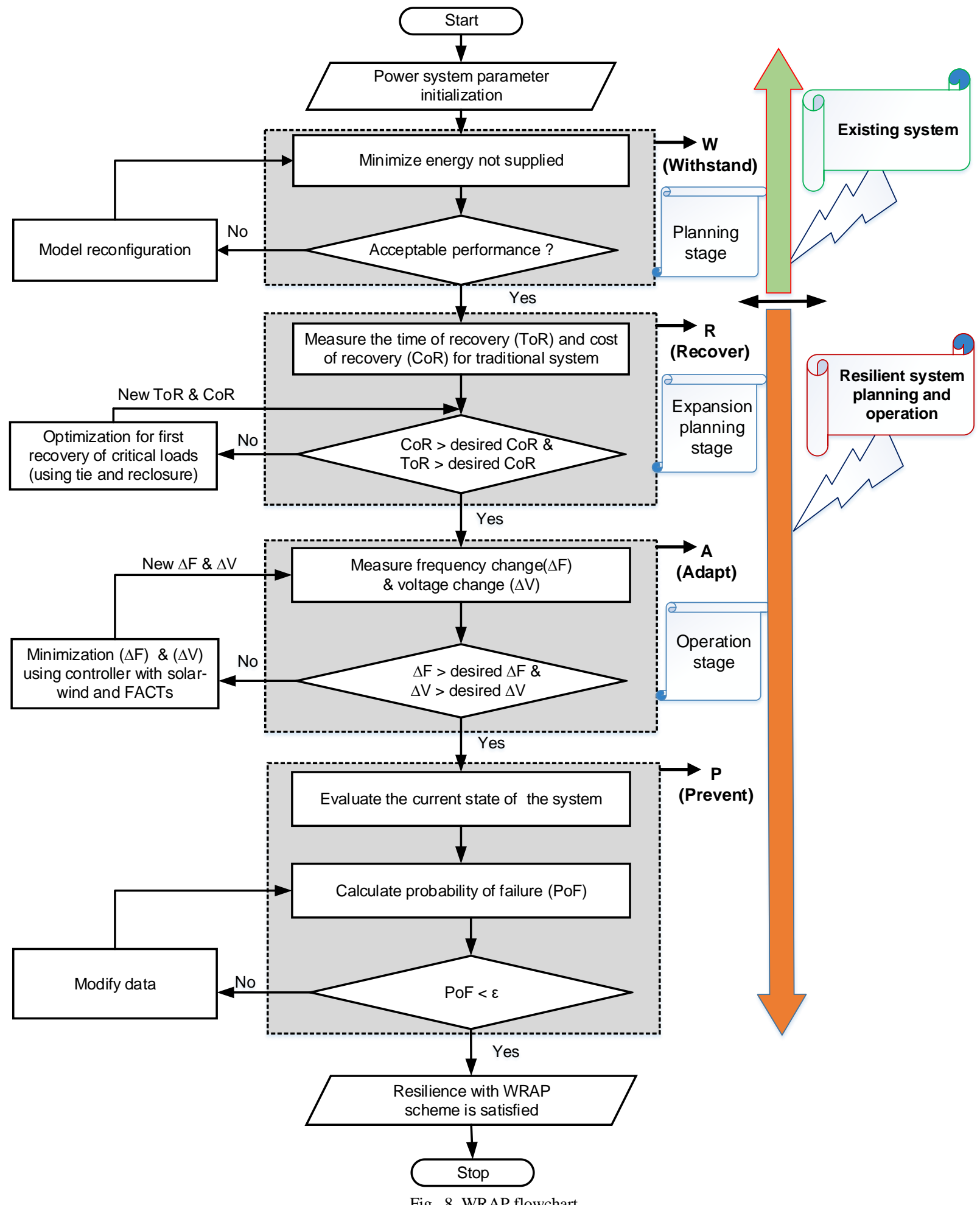

On the basis of the aforementioned discussions, the response of the power systems to inevitable attacks in terms of resilience is a great concern. Therefore, power systems should be prepared for all kinds of unfavorable events through anticipation and decision models [57-59].

\section{WRAP FRAMEWORK}

In this framework, the four elements are used to measure the robustness, rapidity, and probability of response against disruptive events. Moreover, the pre-, during, and post-event planning and operational activities of power systems in the WRAP framework have been speculated, is shown in Fig. 8.
The parameters in the initial attempt include the number of buses, loads, generations and zones. The minimization technique can be used to minimize the energy that is not supplied as a resilience evaluation index. This approach signifies the robustness of the system after the events. If its performance is poor, then the reconfiguration of network is needed to improve the resilience characteristics. Furthermore, the time and cost of recovery can be measured to show the optimal operation of the power system. Meanwhile, a multiobjective optimization method, which can automatically tune the gains of the controller and DG allocation parameters, can 


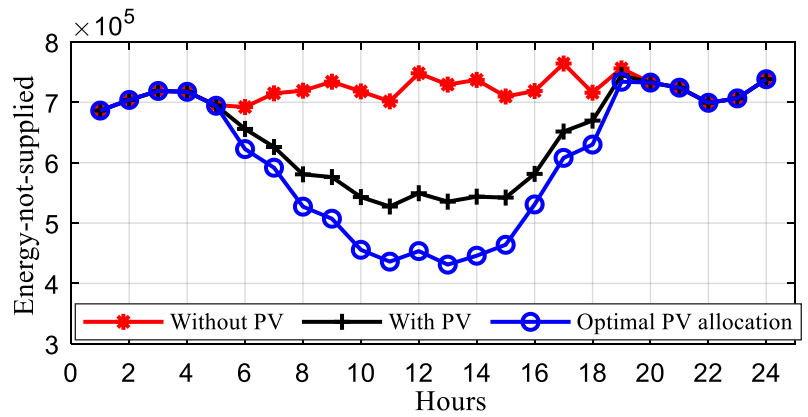

Fig. 9 Energy not supplied in 33-bus system

be adapted to improve the optimal operation and to stabilize the system throughout the events.

Thereafter, a prediction model can be used to predict upcoming events to prepare for and respond to catastrophes. Notwithstanding, this framework aims to provide a conceptual framework to measure the resiliency of the power system. In addition, the use of the WRAP procedure in power systems minimizes the damages and restoration cost and improves its withstanding capabilities.

\section{CASE STUDY}

To support the concept of WRAP, one of the resilience evaluation indexes, i.e., energy-not-supplied is evaluated here through the IEEE-33 bus system with four numbers of PV units. As far as the DG unit is concerned, it is fast becoming a key instrument in the sustainable and resilience power world, which takes a milestone for a modern electric infrastructure towards the value of socio-economic benefits. Mostly the PV and wind units a DG power have been used to meet the load demand during a contingency. Thus, for a case study analysis, authors have installed four PV units in the test system and estimated the energy-not-supplied with and without PV in the response of double fault. Fig. 9 shows the energy-not-supplied in the 33 bus system in 24 hours of duration, where PV with optimal allocation gives a better response than the other two. It is noted that the installation of PV unit in the distribution system, the energy-not-supplied is reduced significantly, which indicates the withstand phase of WRAP and indeed, it shows the resiliency of the system.

\section{CONCLUSION}

The implementation of a resilient structure in the power system network and the enhancement of resilience have become key necessities for the power system. In this paper, available distribution system resilience studies are reviewed and analyzed in accordance with the newly proposed WRAP resilience evaluation framework. The proposed WRAP model can effectively measure the resilience of the power system and enhance its resiliency characteristics in terms of survivability, rapidity, adaptability, and predictability. Furthermore, each element has been described on the basis of the attributes and its resilience evaluation index. This framework contributes in several ways to the understanding of resilience and to providing a basis for resilience measurement. Finally, a case study have demonstrated to support the WRAP framework, which indicates the withstand phase with the objective function as energy-not-supplied and the evidence presented thus far supports the WRAP concept.

Notwithstanding, this topic is a current trend of research; and however, extensive research on this topic needs to be carried out to reinforce the power system network with a remarkable resilience characteristic. Nevertheless, the measurement of resiliency is proposed in the context of the power system by considering each important phase and its corresponding resilience evaluation indices. This model can finally offer the unsupplied energy, time and cost of recovery, and the probability of failure to the users to verify the resilience characteristics.

\section{REFERENCES}

[1] M. J. Ghadi, S. Ghavidel, A. Rajabi, A. Azizivahed, L. Li, and J. Zhang, "A review on economic and technical operation of active distribution systems," Renewable and Sustainable Energy Reviews, vol. 104, pp. 38-53, 2019.

[2] M. J. Ghadi, A. Rajabi, S. Ghavidel, A. Azizivahed, L. Li, and J. Zhang, "From active distribution systems to decentralized microgrids: A review on regulations and planning approaches based on operational factors," Applied Energy, vol. 253, p. 113543, 2019.

[3] Z. Bie, Y. Lin, G. Li, and F. Li, "Battling the extreme: A study on the power system resilience," Proceedings of the IEEE, vol. 105, no. 7, pp. 1253-1266, 2017.

[4] H. Ghoreishi, H. Afrakhte, and M. J. Ghadi, "Optimal placement of tie points and sectionalizers in radial distribution network in presence of DGs considering load significance," in Smart Grid Conference (SGC), 2013, 2013, pp. 160-165: IEEE.

[5] F. H. Jufri, V. Widiputra, and J. Jung, "State-of-the-art review on power grid resilience to extreme weather events: Definitions, frameworks, quantitative assessment methodologies, and enhancement strategies," Applied Energy, vol. 239, pp. 1049-1065, 2019.

[6] J. Wang and H. Gharavi, "Power Grid Resilience [Scanning the Issue]," Proceedings of the IEEE, vol. 105, no. 7, pp. 1199-1201, 2017.

[7] N. Malla, S. Poudel, N. R. Karki, and N. Gyawali, "Resilience of electrical power delivery system in response to natural disasters," in 2017 7th International Conference on Power Systems (ICPS), 2017, pp. 806-811: IEEE.

[8] S. Paul, A. Parajuli, M. Barzegaran, and A. Rahman, "Cyber physical renewable energy microgrid: A novel approach to make the power system reliable, resilient and secure," in 2016 IEEE Innovative Smart Grid Technologies-Asia (ISGT-Asia), 2016, pp. 659-664: IEEE.

[9] S. H. Gilani, H. Afrakhte, and M. J. Ghadi, "Probabilistic method for optimal placement of wind-based distributed generation with considering reliability improvement and power loss reduction," in The 4th Conference on Thermal Power Plants, 2012, pp. 1-6: IEEE.

[10] S. H. Gilani, M. J. Ghadi, and H. Afrakhte, "Optimal allocation of wind turbines considering different costs for interruption aiming at power loss reduction and reliability improvement using imperialistic competitive algorithm," International Review of Electrical Engineering, vol. 8, no. 1, pp. 284-296, 2013.

[11] A. Gholami, T. Shekari, M. H. Amirioun, F. Aminifar, M. H. Amini, and A. Sargolzaei, "Toward a consensus on the definition and taxonomy of power system resilience," IEEE Access, vol. 6, pp. 3203532053, 2018.

[12] H. Haes Alhelou, M. E. Hamedani-Golshan, T. C. Njenda, and P. Siano, "A survey on power system blackout and cascading events: Research motivations and challenges," Energies, vol. 12, no. 4, p. 682, 2019.

[13] S. Ma, S. Li, Z. Wang, and F. Qiu, "Resilience-Oriented Design of Distribution Systems," IEEE Transactions on Power Systems, 2019.

[14] A. K. Srivastava, "Measuring and Enabling Resiliency in Microgrids," presented at the Microgrid Symposiums Bucharest Romania, 2018.

[15] J. Li, X.-Y. Ma, C.-C. Liu, and K. P. Schneider, "Distribution system restoration with microgrids using spanning tree search," IEEE Transactions on Power Systems, vol. 29, no. 6, pp. 3021-3029, 2014.

[16] Z. Wang, B. Chen, J. Wang, and C. Chen, "Networked microgrids for self-healing power systems," IEEE Transactions on smart grid, vol. 7, no. 1, pp. 310-319, 2015.

[17] C. Yuen, A. Oudalov, and A. Timbus, "The provision of frequency control reserves from multiple microgrids," IEEE Transactions on Industrial Electronics, vol. 58, no. 1, pp. 173-183, 2010.

[18] F. Dörfler, J. W. Simpson-Porco, and F. Bullo, "Breaking the hierarchy: Distributed control and economic optimality in microgrids," IEEE Transactions on Control of Network Systems, vol. 3, no. 3, pp. 241-253, 2015.

[19] V. N. Coelho, M. W. Cohen, I. M. Coelho, N. Liu, and F. G. Guimarães, "Multi-agent systems applied for energy systems integration: State-of- 
the-art applications and trends in microgrids," Applied energy, vol. 187, pp. 820-832, 2017.

[20] T. Lv and Q. Ai, "Interactive energy management of networked microgrids-based active distribution system considering large-scale integration of renewable energy resources," Applied Energy, vol. 163, pp. 408-422, 2016.

[21] P. Kou, D. Liang, and L. Gao, "Distributed EMPC of multiple microgrids for coordinated stochastic energy management," Applied energy, vol. 185, pp. 939-952, 2017.

[22] A. Hussain, V.-H. Bui, and H.-M. Kim, "Optimal operation of hybrid microgrids for enhancing resiliency considering feasible islanding and survivability," IET Renewable Power Generation, vol. 11, no. 6, pp. 846-857, 2017

[23] T. Jin, N. Mai, Y. Ding, L. Vo, and R. Dawud, "Planning for Distribution Resilience under Variable Generation: Prevention, Surviving and Recovery," in 2018 IEEE Green Technologies Conference (GreenTech), 2018, pp. 49-56: IEEE.

[24] M. Panteli, D. N. Trakas, P. Mancarella, and N. D. Hatziargyriou, "Boosting the power grid resilience to extreme weather events using defensive islanding," IEEE Transactions on Smart Grid, vol. 7, no. 6 , pp. 2913-2922, 2016

[25] S. Ma, B. Chen, and Z. Wang, "Resilience enhancement strategy for distribution systems under extreme weather events," IEEE Transactions on Smart Grid, vol. 9, no. 2, pp. 1442-1451, 2016.

[26] A. Cárcamo-Gallardo, L. García-Santander, and J. E. Pezoa, "Greedy reconfiguration algorithms for medium-voltage distribution networks," IEEE Transactions on Power Delivery, vol. 24, no. 1, pp. 328-337, 2008

[27] J. Kazempour, P. Pinson, and B. F. Hobbs, "A stochastic market design with revenue adequacy and cost recovery by scenario: Benefits and costs," IEEE Transactions on Power Systems, vol. 33, no. 4, pp. 35313545, 2018.

[28] B. Chen, Z. Ye, C. Chen, J. Wang, T. Ding, and Z. Bie, "Toward a Synthetic Model for Distribution System Restoration and Crew Dispatch," IEEE Transactions on Power Systems, vol. 34, no. 3, pp. 2228-2239, 2018

[29] N. Safaei, D. Banjevic, and A. K. Jardine, "Workforce planning for power restoration: an integrated simulation-optimization approach," IEEE Transactions on Power Systems, vol. 27, no. 1, pp. 442-449, 2011.

[30] J. Q. Tortós and V. Terzija, "Controlled islanding strategy considering power system restoration constraints," in 2012 IEEE Power and Energy Society General Meeting, 2012, pp. 1-8: IEEE.

[31] H. Ahmadi, A. Alsubaie, and J. R. Martí, "Distribution system restoration considering critical infrastructures interdependencies," in 2014 IEEE PES General Meeting| Conference \& Exposition, 2014, pp. 1-5: IEEE.

[32] M. Ibrahim, J. Chen, and R. Kumar, "A resiliency measure for electrical power systems," in 2016 13th International Workshop on Discrete Event Systems (WODES), 2016, pp. 385-390: IEEE.

[33] Y.-P. Fang, C. Fang, E. Zio, and M. Xie, "Resilient Critical Infrastructure Planning Under Disruptions Considering Recovery Scheduling," IEEE Transactions on Engineering Management, 2019.

[34] A. Azizivahed, S. Ghavidel, M. J. Ghadi, L. Li, and J. Zhang, "New energy management approach in distribution systems considering energy storages," in 2017 20th International Conference on Electrical Machines and Systems (ICEMS), 2017, pp. 1-6: IEEE.

[35] M. H. Imani, M. J. Ghadi, S. Ghavidel, and L. Li, "Demand response modeling in microgrid operation: a review and application for incentive-based and time-based programs," Renewable and Sustainable Energy Reviews, vol. 94, pp. 486-499, 2018.

[36] C. Chen, J. Wang, F. Qiu, and D. Zhao, "Resilient distribution system by microgrids formation after natural disasters," IEEE Transactions on smart grid, vol. 7, no. 2, pp. 958-966, 2015.

[37] S. Chanda and A. K. Srivastava, "Defining and enabling resiliency of electric distribution systems with multiple microgrids," IEEE Transactions on Smart Grid, vol. 7, no. 6, pp. 2859-2868, 2016.

[38] M. Nick, R. Cherkaoui, and M. Paolone, "Optimal planning of distributed energy storage systems in active distribution networks embedding grid reconfiguration," IEEE Transactions on Power Systems, vol. 33, no. 2, pp. 1577-1590, 2017

[39] Y. Lin and Z. Bie, "Tri-level optimal hardening plan for a resilient distribution system considering reconfiguration and DG islanding," Applied Energy, vol. 210, pp. 1266-1279, 2018.
[40] A. Kavousi-Fard, M. Wang, and W. Su, "Stochastic resilient posthurricane power system recovery based on mobile emergency resources and reconfigurable networked microgrids," IEEE Access, vol. 6, pp. 72311-72326, 2018

[41] M. H. Imani, K. Yousefpour, M. J. Ghadi, and M. T. Andani, "Simultaneous presence of wind farm and V2G in security constrained unit commitment problem considering uncertainty of wind generation," in 2018 IEEE Texas Power and Energy Conference (TPEC), 2018, pp. 1-6: IEEE.

[42] S. Chanda, A. K. Srivastava, M. U. Mohanpurkar, and R. Hovsapian, "Quantifying Power Distribution System Resiliency Using Code-Based Metric," IEEE Transactions on Industry Applications, vol. 54, no. 4, pp. 3676-3686, 2018.

[43] S. Espinoza, M. Panteli, P. Mancarella, and H. Rudnick, "Multi-phase assessment and adaptation of power systems resilience to natural hazards," Electric Power Systems Research, vol. 136, pp. 352-361, 2016.

[44] S. D. Manshadi and M. E. Khodayar, "Resilient operation of multiple energy carrier microgrids," IEEE Transactions on Smart Grid, vol. 6, no. 5, pp. 2283-2292, 2015.

[45] D. K. Mishra, T. K. Panigrahi, P. K. Ray, and A. Mohanty, "Performance Enhancement of AGC under open market scenario using TDOFPID and IPFC controller," Journal of Intelligent \& Fuzzy Systems, no. Preprint, pp. 1-11, 2018

[46] D. K. MISHRA, T. K. Panigrahi, A. Mohanty, P. K. Ray, and A. K. Sahoo, "Robustness and Stability analysis of Renewable Energy Based Two Area Automatic Generation Control," International Journal of Renewable Energy Research (IJRER), vol. 8, no. 4, pp. 1951-1961, 2018

[47] D. N. Trakas, M. Panteli, N. D. Hatziargyriou, and P. Mancarella, "Spatial risk analysis of power systems resilience during extreme events," Risk Analysis, vol. 39, no. 1, pp. 195-211, 2019.

[48] M. Panteli, P. A. Crossley, D. S. Kirschen, and D. J. Sobajic, "Assessing the impact of insufficient situation awareness on power system operation," IEEE Transactions on power systems, vol. 28, no. 3, pp. 2967-2977, 2013

[49] R. Eskandarpour, A. Khodaei, and A. Arab, "Improving power grid resilience through predictive outage estimation," in 2017 North American Power Symposium (NAPS), 2017, pp. 1-5: IEEE.

[50] R. Eskandarpour and A. Khodaei, "Machine learning based power grid outage prediction in response to extreme events," IEEE Transactions on Power Systems, vol. 32, no. 4, pp. 3315-3316, 2016.

[51] H. Zhang, H. Yuan, G. Li, and Y. Lin, "Quantitative Resilience Assessment under a Tri-Stage Framework for Power Systems," Energies, vol. 11, no. 6, p. 1427, 2018.

[52] S. Yao, P. Wang, and T. Zhao, "Transportable energy storage for more resilient distribution systems with multiple microgrids," IEEE Transactions on Smart Grid, vol. 10, no. 3, pp. 3331-3341, 2018.

[53] N. Perrier et al., "A survey of models and algorithms for emergency response logistics in electric distribution systems. Part I: Reliability planning with fault considerations," Computers \& Operations Research, vol. 40, no. 7, pp. 1895-1906, 2013.

[54] C. Zapata, S. Silva, H. Gonzalez, O. Burbano, and J. Hernandez, "Modeling the repair process of a power distribution system," in 2008 IEEE/PES Transmission and Distribution Conference and Exposition: Latin America, 2008, pp. 1-7: IEEE.

[55] S. Chanda and A. K. Srivastava, "Quantifying resiliency of smart power distribution systems with distributed energy resources," in 2015 IEEE 24th International Symposium on Industrial Electronics (ISIE), 2015, pp. 766-771: IEEE.

[56] M. Panteli, C. Pickering, S. Wilkinson, R. Dawson, and P. Mancarella, "Power system resilience to extreme weather: fragility modeling, probabilistic impact assessment, and adaptation measures," IEEE Transactions on Power Systems, vol. 32, no. 5, pp. 3747-3757, 2016.

[57] C. Wang, Y. Hou, F. Qiu, S. Lei, and K. Liu, "Resilience enhancement with sequentially proactive operation strategies," IEEE Transactions on Power Systems, vol. 32, no. 4, pp. 2847-2857, 2016.

[58] A. Rajabi, L. Li, J. Zhang, J. Zhu, S. Ghavidel, and M. J. Ghadi, "A review on clustering of residential electricity customers and its applications," in 2017 20th International Conference on Electrical Machines and Systems (ICEMS), 2017, pp. 1-6: IEEE.

[59] M. J. Ghadi, S. H. Gilani, A. Sharifiyan, and H. Afrakhteh, "A new method for short-term wind power forecasting," in 2012 Proceedings of 17th Conference on Electrical Power Distribution, 2012, pp. 1-6: IEEE. 